\title{
Analyse alchimique d'orphée \\ de Jean Cocteau
}

Orphêe de Jean Cocteau date de 1925. Cette pièce marque une phase mystique du poète. Rappelons que c'est à la veille de Noël 1925 que Cocteau a pris la communion à Notre-Dame. En effet, c'est après sa cure de désintoxification que Cocteau s'est tourné vers le christianisme. C'est à travers Orphée que Cocteau va questionner la valeur du surréalisme mais surtout le rôle du poète. Il se sert de thème d'Orphée qui représente le mythe de l'évasion, de la beauté en lutte avec la laideur du monde. C'est la recherche de 1'ailleurs, de Dieu. Il est important, avant de nous avancer dans notre étude, de définir ce qu'est le mythe. Pour Jung, ce mythe représente "la somme d'éléments anciens transmis par 1a tradition, traitant de dieux et d'êtres divins, de combat, de héros et de descentes aux Enfers, éléments contenus dans des récits connus mais qui n'excluent pas, cependant, un modelage plus poussé."1 Pour Jung, les mythes sont une création de l'inconscient collectif. Ils représentent des acquisitions permanentes de $I^{\prime}$ esprit et sont, en fait, le symbole d'une réalité fondamentale.

Si nous partons de cette définition, nous voyons que, pour Cocteau, I'amour, la mort, la quête d'Eurydice se chargent d'une signification dont les événements légendaires du mythe ne sont que le revêtement symbolique. Orphée, d'après le mythe, est le prototype, I'image archétype de 1'initiateur, du guide, du prophète inspiré, ce qui, pour Cocteau, revient à dire le poète.

L'auteur résume le sujet de sa pièce ainsi: "Orphée, le grand poète de Thrace, passait pour charmer les fauves. Or il venait de charmer une 
jeune fille, Eurydice, de $1^{\prime}$ arracher au mauvais milieu des Bacchantes. La reine des Bacchantes, furieuse, empoisonna la jeune femme. Orphée obtint d'aller la chercher aux Enfers, mais le pacte lui interdisait de se retourner vers elle; s'il se retournait, il la perdrait pour toujours. I1 se retourna. Les Bacchantes I'assaillirent et $1 \mathrm{e}$ décapitèrent, et, décapité, sa tête appelait encore Eurydice."2 Ce que Cocteau nous invite à considérer, en fait, c'est le drame du poète et de sa souffrance. C'est Ià que Cocteau, en utilisant le mythe, va s'exprimer lui-même et nous faire part de son désarroi moral.

La pièce, du point de vue alchimique, se terminera en sublimatio. En examinant de près les personnages et les scènes, nous établirons comment Orphée peut être considéré comme une oeuvre alchimique où la prima materia subira les transformations nécessaires pour atteindre la pierre philosophale, c'est-à-dire la création poétique, permettant ainsi, grâce à la sensibilité, de faire du poète le messager de 1 'univers.

Dans un prologue, Jean Cocteau nous avertit que sa pièce est un drame qu'il convient d'observer avec attention, car elle représente un exercice périlleux. En effet, tout ici aura un sens. Le décor même fera partie intégrante du drame. Le salon d'Orphée appelle le surnaturel. La scène du thêâtre sera le lieu de rencontre du visible et de l'invisible. Chaque détail aura une signification. C'est la vérité poétique qui transfigurera la vérité. Cocteau nous présente le monde visible comme un monde quotidien, terne. Nous assistons, dès le début, à une scène de ménage entre Orphée et Eurydice. On peut considérer que, symboliquement, cette scène de ménage est la projection de la vie intérieure et de la crise que traverse le poète. Bref, elle annonce déjà la lutte qu'il devra livrer pour accéder au monde invisible, c'est-à-dire atteindre la véritable création poètique. 
Nous pouvons envisager, sans grande difficulté, qu'Orphée et Eurydice représentent la dualité du poète, c'est-à-dire $1^{\prime}$ esprit et $I^{\prime}$ instinct. En fait, Eurydice peut être vue comme étant I'anima du poète. Alchimiquement, Orphée sera soufre. Quoi de plus approprié? N'est-il pas, en effet, le chantre du Soleil? Quant à Eurydice, la femme, elle représente I'obstacle au libre développement de $I^{\prime}$ homme. Elle est vif-argent et là aussi cela représente un choix approprié, car nous savons qu'elle était sous l'influence d'Aglaonice et des Bacchantes qui se consacraient au culte de la Lune. La première scène nous introduit dans une situation, fixatio, qui devient de plus en plus instable. L'atmosphère sera citrinitas. La querelle entre Orphée et Eurydice a pour objet un cheval blanc qui a suivi Orphée et a élu domicile dans l.a maison, au milieu du salon, dans une niche. Le salon est $1^{\prime}$ athanor qui permettra le processus alchimique. Cet animal, envoyé par les muses, est la source vivante de la poésie. Le cheval symbolise le mâle inconscient et, en $I^{\prime}$ occurrence, représente ici le mouvement dada qui, nous le savons, puisait son inspiration dans 1'écriture automatique et $I^{\prime}$ inconscient et aspirait à entrer dans l'univers des rêves. Le cheval sera le catalyste qui précipitera la transformation. On remarquera que le cheval reste à moitié dans 1'ombre. En effet, ce cheval qui révèle les profondeurs de I'inconscient--où se trouvent des trésors enfouis-ne doit pas prendre trop de place. C'est parce que, s'il devient la seule préoccupation du poète, il devient une influence satanique, une tentation où I'inconscient, à travers le rêve, supplante la réalité et devient alors la vraie substance. Le cheval représente la primière tentative d'évasion du poète. La première scène se termine lorsqu'Orphée brise une vitre. Cet acte annonce le changement du stade fixatio vers la première transformation. Entre en scène Heurtebise, vitrier qui est également ange gardien, bien qu'Orphée et Eurydice 
l'ignorent. Les vitres sur son dos représentent les ailes, mais alchimiquement le verre est associé au corps glorifié et au sel. Le verre, qui peut être liquide, indique la possibilité qu'Heurtibise soit un être surhumain, voire, qu'il soit l'incarnation humaine de Dieu. Il est là pour aider Eurydice à triompher du cheval. Il est le sel de notre réaction alchimique, car il sera le conciliateur qui permettra le rapprochement d'Eurydice et d'Orphée. $C^{\prime}$ est lui qui orientera le sacrifice et la réconciliation.

$\mathrm{Au}$ cours des scènes qui suivent, nous apprenons qu'Aglaonice, reine des Bacchantes, s'était opposée au mariage d'Eurydice et d'Orphée et, en plus, elle méprisait les artistes. Elle a accepté d'aider Eurydice à se débarrasser du cheval. Mais ce n'est pas par gentillesse. Aglaonice veut tuer le cheval qui est le symbole de 1'originalité d'Orphée. Elle a donc fait parvenir, par l'entremise d'Heurtebise, un sucre empoisonné et, également, une lettre qui sera $l^{\prime}$ instrument de la perte d'Eurydice.

Dans la scène IV, Orphée, revenu pour prendre son acte de naissance (dont il a besoin pour s'inscrire au concours), s'empare de la chaise sur laquelle se tenait Heurtebise. Celui-ci reste suspendu dans les airs. Orphée qui croit au miracle ne s'en aperçoit pas, alors qu'Eurydice--porteparole des simples mortels--voit le prodige et questionne Heurtebise. E1le dit: "Le mystère est mon ennemi; je me suis décidée à le combattre." Heurtebise part et Eurydice, qui a lêché l'enveloppe, sait qu'elle a été empoisonnée. Elle rappelle Heurtebise et lui demande de ramener Orphée.

Dans la scène VI, la mort entre par le miroir, la porte par laquelle elle va et vient. Pour Cocteau, le miroir est le passage du monde visible au monde invisible. C'est aussi le symbole alchimique qui permet aux fantômes d'apparaître à ceux qui regardent le miroir. La mort, personnifiée en femme 
du monde, est en même temps 1'image du savant moderne, objectif, qui ne fait preuve d'aucun sentiment ou d'émotion. E1le est accompagnée par ses anges Raphaël et Azraël, tous les deux habilés en chirurgien. La mort a été dépouillée de son étrangeté. Elle est assimilée au connu; elle se présente à l'image de la société, car elle fait partie de la vie. Eurydice, qui a souffert, meurt pour Orphée, et le cheval retourne à $1^{\prime}$ univers d'où il est sorti. La colombe, qui est relâchée par la mort, représente $1^{\prime a ̂ m e ~ d ' E u r y d i c e ~ q u i ~ s ' e n v o l e ~ e t ~ s e ~ d e ́ t a c h e ~}$ des ténèbres. Toute I'action mécanique et scientifique de la mort a lieu dans un temps relatif. La mort transforme tout, même le temps qui est mis en valeur par l'emprunt de la montre d'un spectateur et la répétition de la scène.

Orphée, de retour avec Heurtebise, trouve Eurydice morte; le cheval ne l'intéresse plus alors. II abandonne cette possibilité créatrice; il doit retrouver Eurydice et, pour cela, demande I'aide d'Heurtebise. Il va se rendre au royaume de la mort pour reprendre sa femme. Symboliquement, le génie du poète part à la recherche de sa vocation. Cela se fera à l'aide des gants oubliés par la mort et qui rattachent le monde matériel à $1^{\prime}$ inconnu. Orphée devra traverser le miroir. Le génie du poète va se rendre vers l'éternité pour la rejoindre, c'est-à-dire, pour rejoindre le moi du poète. Nous avons ici une atmosphère nigredo et sommes au stade putrefactio de notre analyse alchimique. Le poète obtient la permission de ramener Eurydice, à condition de ne plus jamais la regarder. Heurtebise est toujours là, car son travail $n$ 'est pas fini. Il est convié à rester à déjeuner; mais le repas ne se fera pas, car la transformation $n$ 'est pas encore complète. Nous sommes à l'état separatio. En effet, Eurydice veut vivre en paix avec Orphée, c'est-àdire, conformément à la ligne d'inspiration; Orphée, cependant, n'a pas atteint la pureté d'inspiration qu'il cherche. En fait, ils sont ensemble sans être 
présents I'un à I'autre; ils ne peuvent se toucher. L'union alchimique ne peut avoir lieu à ce stade. Ils se querellent et au cours de leur querelle le mot "lune" revient à plusieurs reprises. L'inévitable arrive; Orphée, par mégarde, regarde Eurydice et celle-ci disparaitt.

Orphée va devoir mourir également pour retrouver dans la mort et la souffrance Eurydice morte pour lui et rétablir ainsi un équilibre avec luimême.

Il ne peut plus utiliser le miroir. Il va devoir mourir et, par sa mort réelle, accéder enfin au chef-d'oeuvre symbolisé dans la pièce par la tête séparée du corps: la raison n'est plus nécessaire. Il choisit sa mort; il marche au martyr. Il est décapité par les Bacchantes quị, poussées par Aglaonice, 1 'accusent d'avoir envoyer un mot injurieux au jury du concours. Cette décapitation, ou démembrement, représente une étape nécessaire à la transformation finale. Eurydice devient alors dans la mort le guide initiatique. Orphée va enfin atteindre la pureté d'inspiration et communier avec le cosmos. La mort d'Orphée procède d'une nécessité spirituelle, de sa volonté de consécration.

Dans la scène XI, Heurtebise reste avec la tête d'Orphée devant le commissaire de police et le greffier. Nous sommes encore au niveau de la terre, nigredo. Par un renversement dû à une éclipse qu'on peut interpréter comme l'union de la lune et du soleil, la foule qui voulait la mort d'Orphée veut maintenant 1'adorer. Le commissaire et le greffier représentent I'humanité sourde et aveugle au signe de l'invisible. Ils ne voient pas Heurtebise qui va rejoindre le couple. Ils ne comprennent pas la disparition du corps d'Orphée.

Dans la scène finale, la maison devient ciel. Orphée, Eurydice et Heurtebise sont pareils mais transformés: remarquons ici le chiffre trois, symbole de la trinité. Ils sont à table; ils communient enfin après avoir traversé, dans I'angoisse, 
des ténèbres redoutables; ils sont arrivés à la découverte de la lumière. Ils sont passés du nigredo à 1 'albedo. Leur aventure a été le passage, à travers $1 e$ sacrifice, du ménage infernal au ménage sanctifié. Dans I'âme du poète il existe un élément femelle et un élément mâle dont $1^{\prime}$ union par un procédé alchimique donne naissance à 1'art. L'amour d'Orphée est l'élément masculin; le génie du poète part à la recherche du principe féminin, la vocation. Il faut que cet amour passe par des épreuves pour parvenir à la réconciliation. Cet amour représente $1^{\prime}$ antidote de la mort.

Orphée, à la recherche d'Eurydice, la vérité poétique, est à la fois initiateur et initié. I1 retrouve ainsi le sens de sa vocation, le sens du rée1. La pièce se termine donc en sublimatio.

CHARLINE SACKS

QUEENS COLLEGE

CITY UNIVERSITY OF NEW YORK

\section{NOTES}

${ }^{1}$ Carl Jung et C. Kerenyi, Introduction à I'essence de la mythologie (Paris: Payot, 1953), p. 11.

2 Jean Cocteau, La Jeunesse et le scandale, dans Oeuvres, IX (Genève: Marguerat, 1950), 345. 